\title{
Improved time to exhaustion following ingestion of the energy drink Amino Impact ${ }^{\mathrm{TM}}$
}

\author{
Allyson L Walsh, Adam M Gonzalez, Nicholas A Ratamess, Jie Kang and Jay R Hoffman*
}

\begin{abstract}
Background: The purpose of this study was to examine the effect of a commercially available energy drink on time to exhaustion during treadmill exercise. In addition, subjective measures of energy, focus, and fatigue were examined

Methods: Fifteen subjects ( 9 men and 6 women; $20.9 \pm 1.0 \mathrm{y} ; 172.1 \pm 9.1 \mathrm{~cm} ; 71.0 \pm 9.4 \mathrm{~kg} ; 16.9 \pm 9.7 \%$ body fat) underwent two testing sessions administered in a randomized, double-blind fashion. Subjects reported to the laboratory in a 3-hr post-absorptive state and were provided either the supplement (SUP; commercially marketed as Amino Impact $\left.{ }^{\mathrm{TM}}\right)$ or placebo (P). During each laboratory visit subjects performed a treadmill run $\left(70 \% \mathrm{VO}_{2}\right.$ max) to exhaustion. Mean $\mathrm{VO}_{2}$ was measured during each endurance exercise protocol. Subjects were required to complete visual analog scales for subjective measures of energy, focus and fatigue at the onset of exercise (PRE), 10-mins into their run (EX10) and immediately post-exercise (IP).

Results: Time to exhaustion was significantly greater $(p=0.012)$ during SUP than P. Subjects consuming the supplement were able to run $12.5 \%$ longer than during the placebo treatment. Subjects consuming SUP reported significantly greater focus $(p=0.031)$, energy $(p=0.016)$, and less fatigue $(p=0.005)$ at PRE. Significant differences between groups were seen at EX10 for focus $(p=0.026)$ and energy $(p=0.004)$, but not fatigue $(p=0.123)$. No differences were seen at IP for either focus ( $p=0.215)$, energy $(p=0.717)$ or fatigue $(p=0.430)$.

Conclusions: Results of this study indicate that the supplement Amino Impact ${ }^{\mathrm{TM}}$ can significantly increase time to exhaustion during a moderate intensity endurance run and improve subjective feelings of focus, energy and fatigue.
\end{abstract}

\section{Introduction}

The use of pre-exercise energy drinks has become a popular supplementation habit among recreational and competitive athletic populations. Recent studies have indicated that among American adolescents and young adults energy drinks are second only to multivitamins in popularity [1,2], with reports suggesting that $30 \%$ of this population group regularly consumes energy drinks [2]. Energy drinks are reported to be quite popular within athletic populations as well $[1,3,4]$. Petroczi and colleagues [4] reported that more than $40 \%$ of British athletes self-admitted to using energy drinks to enhance their workouts or performance. Another study indicated that $89 \%$ of athletes competing in the Ironman World Triathlon Championships admitted that they were planning on using caffeinated supplements prior to competition

\footnotetext{
*Correspondence: hoffmanj@tcnj.edu

1 The College of New Jersey, Ewing, NJ 08628-0718, USA

Full list of author information is available at the end of the article
}

[3]. Athletes from across the performance spectrums (endurance athletes to strength/power athletes) consume energy drinks. However, it is not known whether one type of athlete consumes energy drinks more frequently than another. Anecdotally, it appears that certain energy drinks may be more common for the endurance athlete, while others may be marketed more to the strength/ power athlete. This may be partly attributed to the widely reported benefits that caffeine, an ingredient common in energy drinks, has on endurance performance but not on anaerobic performance [5-11].

Caffeine has been shown to be an effective ergogenic agent by delaying fatigue and increasing time to exhaustion during endurance exercise [5-9]. Its efficacy as an ergogenic aid during anaerobic exercise and strength/ power events though is limited $[8,10,11]$. Recent studies have examined energy drinks that have been marketed primarily to the strength/power athlete [12,13]. These studies investigating a pre-exercise drink comprised of 
caffeine in combination with taurine, glucuronolactone, and branched chain amino acids (BCAA) reported significant improvements in the volume of training (expressed as number of repetitions performed during a bout of resistance exercise) when these supplements were consumed 10 minutes prior to the training session. The greater number of repetitions performed during the training session were associated with a greater anabolic response (elevations in growth hormone) [12].

Recently, a new energy drink has been developed using ingredients similar to those previously discussed studies showing enhanced resistance exercise performance. Considering that many of the ingredients within the energy supplements marketed to the strength/power athlete are similar to that found in supplements used for the endurance athlete, it is of interest to determine whether the ergogenic benefits cross performance spectrums. Interestingly, previous studies that have shown efficacy of a specific energy supplement for one mode of exercise (e.g., endurance exercise) have failed to see similar efficacy in a different exercise protocol (e.g. resistance exercise) [8]. Thus, the purpose of this study is to examine the acute effects of a pre-exercise energy supplement using ingredients previously demonstrated to enhance resistance training performance on time to exhaustion during treadmill exercise, and on subjective feelings of focus, energy and fatigue in healthy, physically active college-aged men and women.

\section{Methods}

\section{Subjects}

Fifteen recreationally active subjects $(9$ men and 6 women; $20.9 \pm 1.0 \mathrm{y} ; 172.1 \pm 9.1 \mathrm{~cm} ; 71.0 \pm 9.4 \mathrm{~kg} ; 16.9 \pm$ 9.7\% body fat) underwent two testing sessions administered in a randomized and double-blind fashion. Subjects were recruited from The College of New Jersey through announcements in the Health and Exercise Science Department. Following an explanation of all procedures, risks, and benefits associated with the experimental protocol, each subject gave his/her written consent prior to participating in this study and completed a medical history/physical activity questionnaire to determine eligibility. Subjects who were pregnant, smokers, taking any medication, had any known metabolic or cardiovascular disease, and/or psychiatric disorder were excluded from the study. Subjects were also required to have been free of any nutritional supplements or ergogenic aids for 6 weeks preceding the study, and were asked to refrain from taking any additional supplement(s) during the course of the study.

\section{Study Design}

The study followed a double-blind, crossover design. Subjects reported to the Human Performance Laboratory on three separate occasions. During the first session subjects performed a maximum oxygen consumption $\left(\mathrm{VO}_{2} \mathrm{max}\right)$ test. During the subsequent two testing session's subjects performed the experimental trials at a standardized time of day. Each testing session was separated by approximately one week $(8.4 \pm 2.2$ days). Subjects were instructed to refrain from consuming any caffeine products on the day of each testing session and from performing any strenuous physical activity for the previous 12 hours. In addition, subjects were instructed to be at least 3 hours post-absorptive state prior to each trial. During each visit to the laboratory subjects were seated for $10 \mathrm{~min}$. Following this resting period subjects were randomly provided either the energy drink supplement (SUP) or a placebo (P). The supplement was provided according to the manufacturer's serving recommendation (26 $\mathrm{g}$ of Amino Impact ${ }^{\mathrm{mm}}$ mixed in 500 $\mathrm{ml}$ of water). On the subject's second visit to the laboratory they were provided with the opposite treatment.

\section{$\mathrm{VO}_{2} \max$ Test}

The $\mathrm{VO}_{2}$ max test was conducted on a treadmill (Desmo model, Woodway, Waukesha, WI) and followed an incremental testing protocol. Briefly, this protocol required the subject to begin exercise at a self-selected speed between 134 and $188 \mathrm{~m} \cdot \mathrm{min}^{-1}$. For the duration of the test, the selfselected speed was maintained while the treadmill elevation increased by $2 \%$ every 2 minutes. The test was preceded by a 5 min warm-up (self-selected running speed at $0 \%$ grade), and was terminated at volitional exhaustion. Immediately following the warm-up period subjects were fitted with a Medgraphics preVent ${ }^{\text {twx }}$ pneumotach (Medical Graphics Corporation, St. Paul, MN) to measure oxygen uptake $\left(\mathrm{VO}_{2}\right)$ and respiratory exchange ratio (RER) through open-circuit spirometry using a metabolic measurement cart (CPX Ultima ${ }^{\mathrm{mm}}$ series, Medical Graphics Corporation, St. Paul, MN). Machine calibration was performed prior to each session using a 3-liter syringe and calibration gases of known concentration of oxygen and carbon dioxide. $\mathrm{VO}_{2}$, minute ventilation, and RER were obtained continuously. Heart rate was measured during the last $15 \mathrm{~s}$ of each min. The maximal value for $\mathrm{VO}_{2}$ was taken as the average of the two highest consecutive values. To ensure that a true maximal $\mathrm{VO}_{2}$ had been attained, all of the following three criteria were met: subject failing to maintain treadmill elevation (\% grade) for 15 consecutive seconds, an increase in $\mathrm{VO}_{2}$ of less than $100 \mathrm{ml} \cdot \mathrm{min}^{-1}$ despite an increase in workload, and a RER greater than 1.05. A best-fit linear regression equation, in which $\mathrm{VO}_{2}$ was plotted as a function of percent grade was developed for each subject. This equation was then used to determine the percent grade and subjects self-selected 
running velocity that corresponded to $70 \% \mathrm{VO}_{2} \max$ for the subsequent endurance trials.

\section{Time to Exhaustion Test}

Subjects exercised at the workload (velocity and \% grade) that elicited $70 \%$ of their $\mathrm{VO}_{2}$ max on the treadmill. Exercise began 10 min following ingestion of the supplement or placebo. Machine calibration and subject preparation were performed as described above. During exercise $\mathrm{VO}_{2}$ and RER were measured continuously. Time to exhaustion was determined as the time that the subject could no longer maintain exercise intensity and/or reached volitional exhaustion.

\section{Questionnaires}

Subjects were instructed to assess their subjective feelings of focus, energy and fatigue using a $10 \mathrm{~cm}$ visual ana$\log$ scale (VAS). The VAS was assessed immediately before commencing exercise (PRE), following $10 \mathrm{~min}$ of exercise (EX10), and immediately post-exercise (IP). Subjects were asked to assess via a mark their feelings at that time with words anchored at each end of the VAS. Questions were structured as "My level of focus is:", with low and high serving as the verbal anchor representing the extreme ratings. Similarly, "My level of energy is:" was anchored with the verbal cues "low" and "high", while "My level of fatigue:" was anchored with the verbal cues "high" and "low". For fatigue, a higher score indicated less fatigue.

\section{Supplement}

On each visit subjects ingested either the supplement or a placebo. The supplement is commercially marketed as 'Amino Impact ${ }^{\mathrm{Tn}}$ ' (Koach, Sport and Nutrition, Langhorne, PA) and consisted of $26 \mathrm{~g}$ of a powder containing an energy matrix (2.05 g of caffeine, taurine, glucuronolactone), a proprietary amino acid matrix $(7.9 \mathrm{~g}$ of L-leucine, L-isoleucine, L-valine, L-arginine and L-glutamine), $5 \mathrm{~g}$ of di-creatine citrate, and $2.5 \mathrm{~g}$ of $\beta$-alanine and mixed with $500 \mathrm{ml}$ of water. The nutritional composition per serving of the supplement was 40 calories with $0 \mathrm{~g}$ of fat. The placebo consisted of $500 \mathrm{ml}$ of water sweetened with $3 \mathrm{~g}$ of sucarlose (Splenda', McNeil Nutritionals, Fort Washington, PA) and colored with red food coloring (McCormick Red Food coloring, McCormick \& Company Hunt Valley, MD) to make it indistinguishable in appearance. The nutritional composition of the placebo contained no calories.

\section{Statistical Analyses}

Performance data were analyzed using paired student's Ttests. Comparisons of subjects' measures of focus, energy and fatigue were accomplished using a repeated measures analysis of variance. In the event of a significant F-ratio, LSD post-hoc tests were used for pairwise comparisons.
A criterion alpha level of $\mathrm{p} \leq 0.05$ was used to determine statistical significance. All data are reported as mean \pm SD.

\section{Results}

Time to exhaustion was significantly greater $(\mathrm{p}=0.012)$ during SUP than P (Figure 1). Subjects consuming the supplement were able to run $12.5 \%$ longer than when they consumed the placebo. Twelve of the 15 subjects were responders where improved performance during the SUP trial was observed. The magnitude of improvement in the responders ranged from 2.9 to $42.8 \%$. The RER for all subjects was greater than 1.1 at the time of exhaustion. No significant difference in RER between trials was observed.

VAS scores of subjective measures of focus, energy and fatigue are presented in Table 1. Subjects consuming SUP reported significantly greater focus $(p=0.031)$, energy ( $p$ $=0.016)$, and less fatigue $(p=0.005)$ at PRE. Significant differences between groups were seen at EX10 for focus $(\mathrm{p}=0.026)$ and energy $(\mathrm{p}=0.004)$, but not fatigue $(\mathrm{p}=$ $0.123)$. No differences were seen at IP for either focus ( $\mathrm{p}=$ $0.215)$, energy $(p=0.717)$ or fatigue $(p=0.430)$.

\section{Discussion}

The results of this study indicate that an acute ingestion of the pre-exercise supplement Amino Impact ${ }^{\mathrm{min}}$ containing caffeine, taurine, glucuronolactone, creatine, $\beta$-alanine, and the amino acids leucine, isoleucine, valine, glutamine and arginine can enhance time to exhaustion during moderate-intensity endurance exercise. In addition, consumption of this supplement 10-min prior to exercise appears to increase subjective feelings of focus, energy and reduce subjective feelings of fatigue before and during endurance exercise.

The most commonly used ingredient in energy drinks is caffeine. Caffeine has been shown to be an effective ergogenic agent by delaying fatigue and increasing time

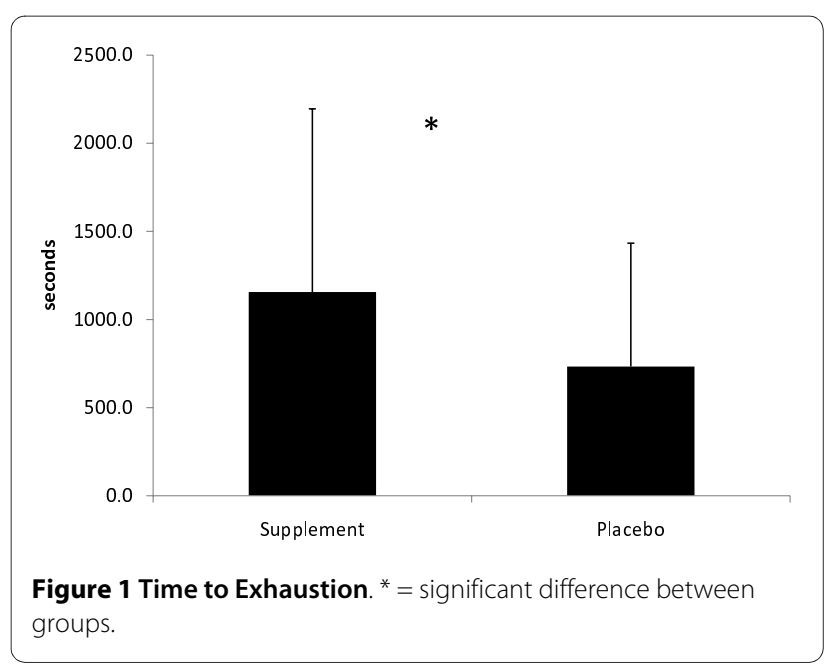


Table 1: Visual Analog Scale Scores for Subjective Measures of Focus, Energy and Fatigue.

\begin{tabular}{|c|c|c|c|c|}
\hline & & Focus & Energy & Fatigue \\
\hline \multirow[t]{2}{*}{ PRE } & SUP & $11.8 \pm 1.9$ & $11.7 \pm 2.0$ & $13.3 \pm 2.8$ \\
\hline & $P$ & $10.5 \pm 2.8^{*}$ & $10.5 \pm 2.8^{*}$ & $11.6 \pm 2.9^{*}$ \\
\hline \multirow[t]{2}{*}{ EX10 } & SUP & $9.9 \pm 3.1$ & $9.7 \pm 2.6$ & $7.3 \pm 3.4$ \\
\hline & $P$ & $7.6 \pm 3.7^{*}$ & $6.1 \pm 3.1^{*}$ & $6.4 \pm 3.3$ \\
\hline \multirow[t]{2}{*}{ IP } & SUP & $5.7 \pm 5.0$ & $3.2 \pm 2.8$ & $1.7 \pm 1.8$ \\
\hline & $P$ & $3.8 \pm 3.6$ & $2.8 \pm 3.3$ & $2.2 \pm 2.2$ \\
\hline
\end{tabular}

to exhaustion during endurance exercise [5-9]. This is thought to be related to caffeine's ability to enhance reliance on fat oxidation preserving muscle glycogen content [14]. Caffeine itself is only a mild central nervous system stimulator [15]. Therefore, additional ingredients (e.g., $\beta$ adrenergic receptor stimulators) are often combined with caffeine to increase the stimulatory response and provide additional ergogenic benefits. The synergistic effect of these ingredients has been demonstrated to increase subjective feelings of alertness, focus and energy [16-19], and improve reaction time to both auditory and visual stimuli [16]. Taurine is often combined with caffeine in energy drinks. Although its mechanism of action is not well understood, previous studies have shown that taurine by itself can improve endurance performance [20,21]. When combined with caffeine and glucuronolactone, ergogenic benefits of taurine have been confirmed in some investigations [12,13], but not others [22]. Differences between these studies may be related to the timing between ingestion and exercise $(10 \mathrm{~min}$ versus $60 \mathrm{~min}$ between consumption and exercise in studies showing a positive response versus a negative response, respectively).

The use of BCAA in energy drinks is becoming more popular. Although BCAAs have been demonstrated to have an important role in protein synthesis [23], and enhance recovery from high-intensity exercise [24], several studies have suggested that BCAA may also improve cognition, focus and psychomotor function [25-28]. Egberts and colleagues [27] reported that BCAA supplementation can improve line tracing, steadiness, attention and auditory reaction time. Most studies demonstrating enhanced cognitive function from BCAA supplementation have been performed on subjects suffering from brain injury $[25,26]$. The mechanism underlying improved cognition has been suggested to be related to changes in amino acid concentrations within the brain [29]. During prolonged physical activity the use of BCAA may counteract or delay fatigue by decreasing the concentration of tryptophan and the synthesis of serotonin $[28,30]$. Serotonin has been implicated as a potential cause of central and mental fatigue during prolonged endurance activity [30], and decreases in this neurotransmitter may have an important role in minimizing or delaying performance decrements during fatiguing exercise. The results of this study suggest a contributory role of the BCAA towards delay in fatigue and enhanced focus. In addition, the combination of both arginine and BCAA has recently been shown to attenuate muscle proteolysis during endurance exercise [31].

The role that creatine may have had on the observed results is not clear. The ergogenic benefits of creatine supplementation have been well-documented [32]. These benefits have been expressed primarily during high intensity exercise and performance in strength/power events following approximately one week of supplementation. Creatine is generally not recognized as a potential ergogenic aid for endurance exercise. However, recent studies have focused on the role that phosphocreatine and the creatine kinase system play in mediating brain and neural function [33,34]. It is thought that $20 \%$ of the body's energy consumption may occur in the brain [33], thus an efficient ATP/PC replenishment system would be critical for normal brain function. Creatine is thought to provide important neuroprotection for the brain through enhancing energy metabolism in brain tissue, promoting antioxidant activities, improving cerebral vasculation (improved brain circulation) and acting as a brain cell osmolyte that can protect the brain against hyper-osmotic shock [35]. Creatine's neuroprotective properties also include stabilization of mitochondrial membranes, stimulation of gluta- 
mate uptake into synaptic vesicles and balance of intracellular calcium homeostasis [36]. These physiological roles for creatine suggest a potential neuroprotective effect that may become important during exhausting exercise. A recent study reported a 7-day loading dose of creatine improved cognitive function, enhanced psychomotor performance and improved mood state during a 36-hour sleep deprivation study [37]. Whether an acute dose of creatine can enhance subjective feelings of focus, energy and fatigue, as indicated by the results of this study, requires further investigation.

The additional ingredients found in Amino Impact ${ }^{\mathrm{Tm}}$ include both glutamine and $\beta$-alanine. Glutamine is a non-essential amino acid that effectively modulates the immune response to exercise and possibly improves athletic performance by enhancing recovery and reducing muscle damage $[38,39]$. A recent investigation has suggested that glutamine may, in part, have an important role in enhancing fluid uptake during endurance exercise under dehydrated conditions [40]. However, its role in enhancing time to exhaustion where no notable hydration stress was present is unknown. It is possible that glutamine preserved hydration levels within the cell, but further research is warranted. Acute $\beta$-alanine supplementation has not been shown to have any role in enhancing endurance performance and likely had no effect in the observed results.

In conclusion, results of this study indicate that the supplement Amino Impact ${ }^{\mathrm{TM}}$ can significantly increase time to exhaustion during a moderate-intensity endurance run. In addition, ingestion of this supplement improved subjective feelings of focus, energy and fatigue at the onset and during the exercise protocol. These results provide evidence that the ingredients of this particular supplement, that have previously been shown to improve acute resistance training performance, can also benefit endurance exercise. This may have important implications as a pre-operation supplement for tactical athletes that are required to perform strength, power and endurance activities as part of their mission objectives.

\section{Competing interests}

Supplement for this project was purchased through Inbounds Athletics. (Denver, CO). All researchers involved collected, analyzed, and interpreted the results from this study. JRH has a financial interest in Koach, Sport and Nutrition. No other author has financial interests concerning the outcome of this investigation. Publication of these findings should not be viewed as endorsement by the investigators, The College of New Jersey or the editorial board of the Journal of International Society of Sports Nutrition.

\section{Authors' contributions}

ALW was the primary investigator, supervised all study recruitment, and data collection. AMG assisted with study recruitment and data collection. JK and NAR were co-authors, oversaw all aspects of study including recruitment, data/ specimen analysis, and manuscript preparation. JRH was involved with study design, statistical analysis, and manuscript preparation. All authors have read and approved the final manuscript.

\section{Acknowledgements}

Authors would like to thank a dedicated group of subjects.

\section{Author Details}

The College of New Jersey, Ewing, NJ 08628-0718, USA

Received: 2 March 2010 Accepted: 15 April 2010

Published: 15 April 2010

\section{References}

1. Froiland K, Koszewski W, Hingst J, Kopecky L: Nutritional supplement use among college athletes and their sources of information. Int J Sports Nutr Exerc Metab 2004, 14:104-120.

2. Hoffman JR, Faigenbaum AD, Ratamess NA, Ross R, Kang J, Tenenbaum G: Nutritional supplementation and anabolic steroid use in adolescents. Med Sci Sports and Exerc 2008, 40:15-24.

3. Desbrow B, Leveritt M: Awareness and use of caffeine by athletes competing at the 2005 Ironman Triathlon World Championships. Int J Sport Nutr Exerc Metab 2006, 16:545-558.

4. Petroczi A, Naughton Dp, Pearce G, Bailey R, Bloodworth A, McNamee MJ: Nutritional supplement use by elite young UK athletes: fallacies of advice regarding efficacy. J Int Soc Sports Nutr 2008, 5:22.

5. Bruce CR, Anderson ME, Fraser SF, Stepto NK, Klein R, Hopkins WG, Hawley JA: Enhancement of 2000-m rowing performance after caffeine ingestion. Med Sci Sports Exerc 32:1958-1963.

6. Graham TE, Hibbert E, Sathasivam P: Metabolic and exercise endurance effects of coffee and caffeine ingestion. J App/ Physiol 1998, 85:883-889.

7. Graham TE, Spriet LL: Performance and metabolic responses to a high caffeine dose during prolonged exercise. J Appl Physiol 1995, 78:867-874.

8. Hoffman JR, Kang J, Ratamess NA, Jennings PF, Mangine G, Faigenbaum AD: Effect of Nutritionally Enriched Coffee Consumption on Aerobic and Anaerobic Exercise Performance. J Strength Cond Res 2007, 21:456-459.

9. Hogervorst E, Bandelow S, Schmitt J, Jentjens R, Oliveira M, Allgrove J, Carter T, Gleeson M: Caffeine improves physical and cognitive performance during exhaustive exercise. Med Sci Sports Exerc 2008, 40:1841-1851.

10. Kalmar JM: The influence of caffeine on voluntary muscle activation. Med Sci Sports Exerc 2005, 37:2113-2119.

11. Woolf K, Bidwell WK, Carlson AG: Effect of caffeine as an ergogenic aid during anaerobic exercise performance in caffeine naïve collegiate football players. J Strength Cond Res 2009:1363-1369.

12. Hoffman JR, Ratamess NA, Ross R, Shanklin M, Kang J, Faigenbaum AD: Effect of a Pre-Exercise 'High-Energy' Supplement Drink on the Acute Hormonal Response to Resistance Exercise. J Strength Cond Res 2008, 22:874-882

13. Ratamess NA, Hoffman JR, Ross R, Shanklin M, Faigenbaum AD, Kang J: Effects of an Amino Acid/Creatine/Energy Supplement on Performance and the Acute Hormonal Response to Resistance Exercise. Int J Sport Nutr Exerc Metab 2007, 17:608-623.

14. Spriet LL: Caffeine and performance. Int J Sport Nutr 1995, 5:S84-S99.

15. Sawynok J: Pharmacological rationale for the clinical use of caffeine. Drugs 1995, 49:37-51.

16. Hoffman JR, Kang J, Ratamess NA, Hoffman MW, Tranchina CP Faigenbaum AD: Examination of a high energy, pre-exercise supplement on exercise performance. J Int Soc Sports Nutr 2009, 6:2.

17. Scholey AB, Kennedy DO: Cognitive and physiological effects of an "energy drink": an evaluation of the whole drink and of glucose, caffeine, and herbal flavouring fractions. Psychopharm 2004, 176:320-330.

18. Smit HJ, Cotton JR, Hughes SC, Rogers PJ: Mood and cognitive performance effects of "energy" drink constituents: caffeine, glucose and carbonation. Nutr Neurosci 2004, 7:127-139.

19. Smith A: Effects of caffeine on human behavior. Food Chem Toxico/ 2002, 40:1243-1255.

20. Miyazaki T, Matsuzaki Y, Ikegami T, Miyakawa S, Doy M, Tanaka N, Bouscarel B: Optimal and effective oral doses of taurine to prolong exercise performance in rat. Amino Acids 2004, 27:291-298.

21. Zhang M, Izuma I, Kagamimori S, Sokejima S, Yamagami T, Liu Z, Qi B: Role of taurine supplementation to prevent exercise-induced oxidative stress in healthy young men. Amino Acids 2004, 26:203-207. 
22. Candow DG, Kleisinger AK, Grenier S, Dorsch KD: Effect of sugar-free red bull energy drink on high-intensity run time-to-exhaustion in young adults. J Strength Cond Res 2009, 23:1271-1275.

23. Tipton KD, Rasmussen BB, Miller SL, Wolf SE, Owens-Stovall SK, Petrini BE, Wolfe RR: Timing of amino acid-carbohydrate ingestion alters anabolic response of muscle to resistance exercise. Am J Phys Endocr Metab 2001, 281:E197-E206.

24. Hoffman JR, Ratamess NA, Tranchina CP, Rashti SL, Kang J, Faigenbaum AD: Effect of Protein Ingestion on Recovery Indices Following a Resistance Training Protocol in Strength/Power Athletes. Amino Acids 2009 in press.

25. Aquilani R, ladarola P, Contardi A, Boselli M, Verri M, Pastoris O, Boschi F, Arcidiaco P, Viglio S: Branched-chain amino acids enhance the cognitive recovery of patients with severe traumatic brain injury. Arch Phys Med Rehab 2005, 86:1729-1735.

26. Cole JT, Mitala CM, Kundu S, Verma A, Elkind JA, Nissim I, Cohen AS: Dietary branched chain amino acids ameliorate injury-induced cognitive impairment. Proc Natl Acad Sci 2010, 107:366-371.

27. Egberts EH, Schomerus H, Hamster W, Jurgens P: Branched chain amino acids in the treatment of latent portosystemic encephalopathy. A double blind placebo controlled crossover study. Gastroenterology 1985, 88:887-895

28. Fernstrom JD: Branched-chain amino acids and brain function. J Nutr 2005, 135:1539s-1546s.

29. Meeusen $R$, Watson P: Amino acids and the brain: do they play a role in "central fatigue"? Int J Sports Nutr Exerc Metab 2007, 17:S37-S46.

30. Davis JM, Alderson NL, Welsh RS: Serotonin and central nervous system fatigue: nutritional considerations. Am J Clin Nutr 2000, 72:573S-578S.

31. Matsumoto K, Mizuno M, Mizuno T, Dilling-Hansen B, Lahoz A, Bertelsen V, Münster $\mathrm{H}$, Jordening $\mathrm{H}$, Hamada K, Doi T: Branched-chain amino acids and arginine supplementation attenuates skeletal muscle proteolysis induced by moderate exercise in young individuals. Int J Sports Med 2007, 28:531-538

32. Hoffman JR, Stout JR: Performance-Enhancing Substances. In Essentials of Strength and Conditioning 3rd edition. Edited by: Earle RW, Baechle TR. Human Kinetics: Champaign, IL; 2008:179-200.

33. Shulman RG, Rothman DL, Behar KL, Hyder F: Energetic basis of brain activity: implications for neuroimaging. Trends Neurosci 2004, 27:489-495

34. Stockler S, Schutz PW, Salomons GS: Cerebral creatine deficiency syndromes: clinical aspects, treatment and pathophysiology. Subcell Biochem 2007, 46:149-66.

35. Andres RH, Ducray AD, Schlattner U, Wallimann T, Widmer HR: Functions and effects of creatine in the central nervous system. Brain Res Bul 2008, 76:329-343.

36. Ellis $A C$, Rosenfeld $\mathrm{J}$ : The role of creatine in the management of amyotrophic lateral sclerosis and other neurodegenerative disorders. CNS Drugs 2004, 18:967-980

37. McMorris T, Harris RC, Howard AN, Langridge G, Hall B, Corbett J, Dicks M, Hodgson C: Creatine supplementation, sleep deprivation, cortisol, melatonin and behavior. Physiol Behav 2007, 90:21-28

38. Castell LM, Newsholme EA: Glutamine and the effects of exhaustive exercise upon the immune response. Can J Physiol Pharmacol 1998, 76:524-532.

39. Favano A, Santos-Silva PR, Nakano EY, Pedrinelli A, Hernandez AJ, Greve $J \mathrm{M}$ : Peptide glutamine supplementation for tolerance of intermittent exercise in soccer players. Clinics 2008, 63:27-32.

40. Hoffman JR, Ratamess NA, Kang J, Rashti SL, Kelly N, Gonzalez AM, Stec M, Andersen S, Bailey BL, Yamamoto LM, Hom LL, Kupchak BR, Faigenbaum $A D$, Maresh CM: Examination of the efficacy of acute L-Alanyl-LGlutamine during Hydration Stress in Endurance Exercise. J Int Soc Sports Nutr 2010, 7:8.

doi: $10.1186 / 1550-2783-7-14$

Cite this article as: Walsh et al., Improved time to exhaustion following

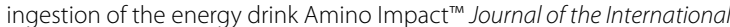
Society of Sports Nutrition 2010, 7:14

\section{Submit your next manuscript to BioMed Central and take full advantage of:}

- Convenient online submission

- Thorough peer review

- No space constraints or color figure charges

- Immediate publication on acceptance

- Inclusion in PubMed, CAS, Scopus and Google Scholar

- Research which is freely available for redistribution 